Discrete Comput Geom 37:455-469 (2007)

DOI: $10.1007 / \mathrm{s} 00454-006-1302-7$

\title{
New Non-Linear Inequalities for Flag-Vectors of 4-Polytopes
}

\author{
Joseph M. Ling \\ Department of Mathematics and Statistics, The University of Calgary, \\ Calgary, Alberta, Canada T2N 1N4 \\ ling@math.ucalgary.ca
}

\begin{abstract}
In this paper we prove four new (infinite) lists of quadratic inequalities, and four cubic inequalities, for the flag $f$-vectors of 4-polytopes. These extend and supplement the only four currently known non-linear inequalities, which were proved by Bayer in 1987. The new lists of inequalities for flag $f$-vectors yield new lists of inequalities for $f$-vectors of 4-polytopes. Using the latter, we managed to improve an estimate discovered by Höppner and Ziegler concerning upper bounds of $f_{1}$ in terms of $f_{0}$ and $f_{3}$.
\end{abstract}

\section{Introduction and Main Results}

Let $P$ be a convex $d$-polytope. The $i$-dimensional faces of $P$ are called the $i$-faces of $P$, $i=-1,0,1,2,3, d$. We understand the unique (-1)-face of $P$ to be the empty set, the 0 -faces the vertices, and the unique $d$-face the polytope $P$ itself. For $i=-1, \ldots, d$, let $f_{i}(P)$ denote the number of $i$-faces of $P$. We define the $f$-vector of $P$ to be

$$
f(P)=\left(f_{0}(P), f_{1}(P), f_{2}(P), \ldots, f_{d-1}(P)\right) .
$$

Let $S=\left\{i_{1}, \ldots, i_{p}\right\}$ be a subset of $\{-1,0,1, \ldots, d\}$ such that $i_{1}<i_{2}<\cdots<i_{p}$. An $S$-flag is an increasing sequence of faces $F_{1} \subset F_{2} \subset \cdots \subset F_{p}$ of $P$ such that $\operatorname{dim} F_{j}=i_{j}$ for $j=1,2, \ldots, p$. We define $f_{S}(P)$ to be the number of $S$-flags of $P$. For simplicity's sake, we often write $f_{013}$ for $f_{\{0,1,3\}}$, etc. The collection of all $f_{S}(P)$ 's is called the flag $f$-vector of $P$. Although the flag $f$-vector has as many components as there are subsets of $\{-1,0,1, \ldots, d\}$, there are plenty of redundancies among the component, so much so that all components are determined by only $c_{d}-1$ of them, where $c_{d}$ is the $d$ th Fibonacci number: $c_{1}=1, c_{2}=2$, and $c_{n+2}=c_{n}+c_{n+1}$. This result is mainly the consequence of the celebrated generalized Dehn-Sommerville relations discovered by 
Bayer and Billera [2]:

$$
\sum_{j=i+1}^{k-1}(-1)^{j-i-1} f_{S \cup\{j\}}=\left(1-(-1)^{k-i-1}\right) f_{S},
$$

where $[i, k] \cap S=\{i, k\}$. With regard to our objects of investigation in this paper, the flag $f$-vectors of 4-polytopes are determined by four of the $f_{S}$ 's such as $\left(f_{0}, f_{1}, f_{2}, f_{02}\right)$, as used in [1], or $\left(f_{0}, f_{1}, f_{3}, f_{13}\right)$, as used in [6]. For the sake of easy reference, the expressions of the flag $f$-vector components in terms of these two bases are listed in Appendix A of this paper.

The classical problems of characterizing the $f$-vectors and the flag $f$-vectors of 4polytopes are far from being solved. No new inequalities have been discovered since Bayer published her list in 1987 [1]. We list it here:

Theorem [1]. If $\left(f_{0}, f_{1}, f_{2}, f_{02}\right)=\left(f_{0}(P), f_{1}(P), f_{2}(P), f_{02}(P)\right)$ for some 4-polytope $P$, then

(1) $f_{02}-3 f_{2} \geq 0$.

(2) $f_{02}-3 f_{1} \geq 0$.

(3) $f_{02}-3 f_{2}+f_{1}-4 f_{0}+10 \geq 0$.

(4) $6 f_{1}-6 f_{0}-f_{02} \geq 0$.

(5) $f_{0}-5 \geq 0$.

(6) $f_{2}-f_{1}+f_{0}-5 \geq 0$.

(7) $2\left(f_{02}-3 f_{2}\right)+f_{1} \leq\left(\begin{array}{c}f_{0} \\ 2\end{array}\right)$.

(8) $2\left(f_{02}-3 f_{1}\right)+f_{2} \leq\left(\begin{array}{c}f_{2}-f_{1}+f_{0} \\ 2\end{array}\right)$.

(9) $f_{02}-4 f_{2}+3 f_{1}-2 f_{0} \leq\left(\begin{array}{c}f_{0} \\ 2\end{array}\right)$.

(10) $f_{02}+f_{2}-2 f_{1}-2 f_{0} \leq\left(\begin{array}{c}f_{2}-f_{1}+f_{0} \\ 2\end{array}\right)$.

To facilitate the analysis of the "fatness" of 4-polytopes, Höppner and Ziegler [6] have put these inequalities in terms of $\left(f_{0}, f_{1}, f_{3}, f_{03}\right)$. We have placed these in Appendix B.

While much more has been discovered for dimensions 5 and higher, techniques that are fruitful for these dimensions fail to yield any new inequalities for 4-polytopes. For dimensions 5 and above, see Kalai's convolution operation [7], and Ehrenborg's technique of lifting inequalities [4], as well as their references to earlier works. For relationships among flag $f$-vector (linear) inequalities, see [10]. See also the (lack of) updates on 4-polytope inequalities in [5], [6], [3], and [8].

Our main goal of this paper is to prove four new infinite lists of non-linear inequalities for flag $f$-vectors of 4-polytopes, extending Bayer's (7) and (8), and supplementing Bayer's (9) and (10). With $\left(f_{0}, f_{1}, f_{2}, f_{02}\right)$ denoting that for a general 4-polyotpe, here are our main results:

Theorem 2.1. For any integer $k$,

$$
(k-1) f_{02}-\left(\begin{array}{c}
k+1 \\
2
\end{array}\right) f_{2}+f_{1} \leq\left(\begin{array}{c}
f_{0} \\
2
\end{array}\right) .
$$

Equality holds only if $k=2,3$, every two vertices of $P$ lie on a common 2-face, and 
each 3-face of $P$ is (combinatorially equivalent to) either the tetrahedron, the pyramid with a square base, or the triangular prism.

Corollary 2.2. For any integer $k \neq 2,3$,

$$
(k-1) f_{02}-\left(\begin{array}{c}
k+1 \\
2
\end{array}\right) f_{2}+f_{1}+1 \leq\left(\begin{array}{c}
f_{0} \\
2
\end{array}\right) .
$$

We note that despite the ranges of $k$ indicated in these results, the only cases of interest have $k \geq 3$. Theorem 2.1 and Corollary 2.2 comprise the first new list of inequalities we prove in this paper. A direct consequence of Theorem 2.1 is the following result, which we conjecture would also imply Theorem 2.1.

\section{Corollary 2.3.}

$$
f_{02}\left(f_{02}-3 f_{2}\right) \leq 2 f_{2}\left[\left(\begin{array}{c}
f_{0} \\
2
\end{array}\right)-f_{1}\right] \text {. }
$$

A second list of inequalities is given in Theorem 2.5:

Theorem 2.5. For any integer $k$,

$$
2(k-1) f_{02}-k(k+1) f_{2}+\left(k^{2}-3 k+4\right) f_{1}-k(k-3) f_{0} \leq 4\left(\begin{array}{c}
f_{0} \\
2
\end{array}\right) .
$$

Equality holds only if every 3-face of $P$ has $k$ or $k+1$ vertices, every two vertices of $P$ lie on a common 2-face, and each 3-face of $P$ is (combinatorially equivalent to) either the pyramid with a $(k-1)$-gon base or the pyramid with a $k$-gon base, or in the case when $k=3$, also the triangular prism.

Bayer's (7) is the special case of Theorem 2.1 where $k=3$. Theorem 2.5 supplements Bayer's (9) in the sense that they come from a slight modification in the proof of Bayer's (9). Yet, many, if not all, of the inequalities in Theorem 2.5 are not weaker than (9). The relative strength of these inequalities are discussed briefly in Section 3. The duals of Theorems 2.1 and 2.5 play corresponding roles for Bayer's (8) and (10), which are the duals of Bayer's (7) and (9). Our third and fourth list of new inequalities, then, are the duals of the two lists headed by Theorems 2.1 and 2.5.

The projections of Bayer's (1)-(10) onto the $f$-vector space yields inequalities for the $f$-vectors $\left(f_{0}, f_{1}, f_{2}, f_{3}\right)$ of 4-polytopes. In terms of $\left(f_{0}, f_{1}, f_{2}\right)$, these inequalities read:

Theorem [1]. If $\left(f_{0}, f_{1}, f_{2}\right)=\left(f_{0}(P), f_{1}(P), f_{2}(P)\right)$ for some 4-polytope $P$, then

(11) $-f_{2}+3 f_{1}-2 f_{0} \leq\left(\begin{array}{c}f_{0} \\ 2\end{array}\right)$.

(12) $f_{2}+f_{1}-2 f_{0} \leq\left(\begin{array}{c}f_{0}-f_{1}+f_{2} \\ 2\end{array}\right)$.

Expressed in terms of $\left(f_{0}, f_{1}, f_{3}\right)$, these become

$$
\begin{aligned}
& \left(11^{\prime}\right)-f_{0}+2 f_{1}-f_{3} \leq\left(\begin{array}{c}
f_{0} \\
2
\end{array}\right) . \\
& \left(12^{\prime}\right)-3 f_{0}+2 f_{1}+f_{3} \leq\left(\begin{array}{c}
f_{3} \\
2
\end{array}\right) .
\end{aligned}
$$


Höppner and Ziegler [6] use $\left(11^{\prime}\right)$ to obtain the inequality

$$
f_{1} \leq\left\lfloor\frac{n^{2}+3 n}{4}\right\rfloor, \quad n=\max \left\{f_{0}, f_{3}\right\},
$$

which throws light on the size of the fatness $\varphi(P):=\left(f_{1}+f_{2}\right) /\left(f_{0}+f_{3}\right)$ of 4 polytopes. (This seems to be the original definition of fatness. The formal definition has subsequently been modified. See, for instance, [11] and [12].) When we project the newly obtained lists of inequalities onto the $f$-vector space, we obtain lists of inequalities, and consequently are able to refine Höppner and Ziegler's result. In particular, we arrive at

$$
f_{1} \leq\left\lfloor\frac{1}{k} n^{2}+\frac{k-1}{2} n\right\rfloor, \quad n=\max \left\{f_{0}, f_{3}\right\},
$$

for all $k \geq 3$. These are presented in Section 4 .

In the appendices we exhibit the known inequalities for 4-polytopes for future reference. We have also chosen to express all results both in terms of the "sparse base" $\left(f_{0}, f_{1}, f_{2}, f_{02}\right)$ and in terms of $\left(f_{0}, f_{1}, f_{3}, f_{03}\right)$. The list can not only be used for easy reference, but also shows how different inequalities take on an elegant form by different choices of base.

\section{Definitions and Proof of the Main Theorems}

For a convex $d$-polytope $Q$, there are $\left(f_{0}(Q)\right)$ pairs of vertices. These vertices fall into $d$ pairwise disjoint classes $\mathcal{M}_{i}(Q), i=1,2, \ldots, d$, where a pair $\{u, v\}$ of vertices belong to $\mathcal{M}_{i}(Q)$ if and only if it is contained in some $i$-face but is not contained in any $(i-1)$-face of $Q$. If $m_{i}(Q)$ denotes the cardinality of $\mathcal{M}_{i}(Q)$, then

$$
\begin{aligned}
m_{1}(Q) & =f_{1}(Q), \\
\sum_{i=1}^{d} m_{i}(Q) & =\left(\begin{array}{c}
f_{0}(Q) \\
2
\end{array}\right) .
\end{aligned}
$$

Below, $F$ denotes a general 2-face, and $G$ a 3-face, of a 4-polytope $P$, and we write $f_{S}$ for $f_{S}(P)$ for any subset $S$ of $\{0,1,2,3\}$.

Our first result is an extension of the first inequality of Bayer. Our proof is a slight adjustment of Bayer's. We also include a condition for equality to hold.

Theorem 2.1. For any integer $k$,

$$
(k-1) f_{02}-\left(\begin{array}{c}
k+1 \\
2
\end{array}\right) f_{2}+f_{1} \leq\left(\begin{array}{c}
f_{0} \\
2
\end{array}\right) .
$$

Equality holds only if $k=2$ or 3 , every two vertices of $P$ lie on a common 2-face, and each 3-face of $P$ is (combinatorially equivalent to) either the tetrahedron, the pyramid with a square base or the triangular prism. 
Proof. Modifying Bayer's calculation at one step, we see that for any 2-face $F$ of $P$,

$$
\begin{aligned}
m_{2}(F) & =\left(\begin{array}{c}
f_{0}(F) \\
2
\end{array}\right)-f_{1}(F) \\
& =\frac{1}{2}\left[f_{0}(F)-k\right]\left[f_{0}(F)-k-1\right]+(k-1) f_{0}(F)-\left(\begin{array}{c}
k+1 \\
2
\end{array}\right) \\
& \geq(k-1) f_{0}(F)-\left(\begin{array}{c}
k+1 \\
2
\end{array}\right) .
\end{aligned}
$$

Summing over all 2-faces of $P$, we have

$$
m_{2} \geq(k-1) f_{02}-\left(\begin{array}{c}
k+1 \\
2
\end{array}\right) f_{2} .
$$

Thus,

$$
(k-1) f_{02}-\left(\begin{array}{c}
k+1 \\
2
\end{array}\right) f_{2}+f_{1} \leq m_{2}+m_{1} \leq\left(\begin{array}{c}
f_{0} \\
2
\end{array}\right) .
$$

Now, if equality holds, then we have $m_{3}=m_{4}=0$, and $f_{0}(F)=k$ or $k+1$ for all 2-faces $F$ of $P$. That $m_{3}=m_{4}=0$ means that every two vertices lie on a common 2 -face. If now there is a 2-face $F$ with five or more vertices, say, $F=\left[v_{1}, v_{2}, \ldots, v_{p}\right]$ whose edges are $\left[v_{i}, v_{i+1}\right], i=1,2, \ldots, p$, where $p \geq 5$ and $v_{p+1}=v_{1}$, we consider a face $F^{\prime}$ adjacent to $F$ within some 3 -face $G$ of $P$. Suppose $F^{\prime}=\left[v_{1}^{\prime}, v_{2}^{\prime}, \ldots, v_{q}^{\prime}\right]$, whose edges are $\left[v_{j}^{\prime}, v_{j+1}^{\prime}\right], j=1,2, \ldots, q$, where $v_{q+1}^{\prime}=v_{1}^{\prime}$, and $v_{1}^{\prime}=v_{1}, v_{q}^{\prime}=v_{p}$. Since each 2-face has either $k$ or $k+1$ vertices, $q$ must be $p-1, p$, or $p+1$. In any case, $q \geq p-1 \geq 4$. Consider the five vertices $v_{2}, v_{3}, v_{4}, v_{2}^{\prime}, v_{q-1}^{\prime}$. Radon's theorem implies that if these vertices are in general position, then some pair of vertices among them will yield an open line segment lying in the relative interior of $G$. This contradicts $m_{3}=0$. Thus, some four of them lie on the same 2-plane $\pi$. However, in order that every pair of these four points lies on a common 2-face, the plane $\pi$ must be the defining plane for that 2-face. Clearly, the four points must contain both $v_{2}^{\prime}$ and $v_{q-1}^{\prime}$, and cannot be $v_{2}, v_{4}, v_{2}^{\prime}, v_{q-1}^{\prime}$. Nonetheless, if the four points are $v_{2}, v_{3}, v_{2}^{\prime}, v_{q-1}^{\prime}$, then $\left[v_{4}, v_{2}^{\prime}\right]$ will fail to lie on a 2 -face. This shows that no 2-face can have five or more vertices. This implies that $k=2,3$, or 4 , and in case $k=4$, all 2-faces have exactly four edges, and in case $k=2$, all 2-faces are triangles.

By a similar argument, we see that if there are two adjacent 2-faces $F_{1}=\left[v_{1}, v_{2}, v_{3}, v_{4}\right]$ and $F_{2}=\left[v_{1}, v_{2}^{\prime}, v_{3}^{\prime}, v_{4}\right]$ in a 3-face $G$ of $P$, then $v_{2}, v_{3}, v_{2}^{\prime}, v_{3}^{\prime}$ must define a 2-face of $G$, and the condition $m_{3}=0$ forces $G$ to be the triangular prism. This rules out the possibility $k=4$. To complete the proof, it remains to see that if $G$ has a quadrilateral 2face adjacent to four triangular 2-faces, then $G$ must be the pyramid with a quadrilateral base.

Corollary 2.2. For any integer $k \neq 2,3$,

$$
(k-1) f_{02}-\left(\begin{array}{c}
k+1 \\
2
\end{array}\right) f_{2}+f_{1}+1 \leq\left(\begin{array}{c}
f_{0} \\
2
\end{array}\right) .
$$




\section{Corollary 2.3.}

$$
f_{02}\left(f_{02}-3 f_{2}\right) \leq 2 f_{2}\left[\left(\begin{array}{c}
f_{0} \\
2
\end{array}\right)-f_{1}\right]
$$

Proof. Rewriting

$$
(k-1) f_{02}-\left(\begin{array}{c}
k+1 \\
2
\end{array}\right) f_{2}+f_{1} \leq\left(\begin{array}{c}
f_{0} \\
2
\end{array}\right)
$$

in the form

$$
Q(k):=f_{2} k^{2}-\left(2 f_{02}-f_{2}\right) k+2\left[\left(\begin{array}{c}
f_{0} \\
2
\end{array}\right)-f_{1}+f_{02}\right] \geq 0,
$$

we see that the roots of the quadratic $Q(k)$ are either complex or real but no more than a unit apart. This translates into an inequality on the discriminant.

Remarks. 1. Bayer's (7) is our case $k=3$.

2. One could also modify the proof of Theorem 2.1 to obtain a continuum of inequalities

$$
\left(k-\frac{3}{2}\right) f_{02}-\frac{k^{2}}{2} f_{2}+f_{1} \leq\left(\begin{array}{c}
f_{0} \\
2
\end{array}\right), \quad k \text { any real number. }
$$

All one needs is the step

$$
\begin{aligned}
m_{2}(F) & =\left(\begin{array}{c}
f_{0}(F) \\
2
\end{array}\right)-f_{1}(F) \\
& =\frac{1}{2}\left[f_{0}(F)-k\right]^{2}+\left(k-\frac{3}{2}\right) f_{0}(F)-\frac{k^{2}}{2} \\
& \geq\left(k-\frac{3}{2}\right) f_{0}(F)-\frac{k^{2}}{2} .
\end{aligned}
$$

However, this gives us nothing new, for the continuum of inequalities is embodied in the single inequality in Corollary 2.3 via a discriminant consideration.

Next, we look at inequalities supplementing Bayer's (9). To do that, we first state a supplementary lemma whose proof we omit, as it is similar to that of Theorem 2.1.

Lemma 2.4. Given any 3-polytope $G$, and any integer $k$,

$$
m_{2}(G)+m_{3}(G) \geq k f_{0}(G)-f_{1}(G)-\left(\begin{array}{c}
k+1 \\
2
\end{array}\right) .
$$

Theorem 2.5. For any integer $k$,

$$
f_{1}+\frac{1}{2}\left\{k f_{03}-f_{13}-\left(\begin{array}{c}
k+1 \\
2
\end{array}\right) f_{3}\right\} \leq\left(\begin{array}{c}
f_{0} \\
2
\end{array}\right)
$$


or, in terms of $\left(f_{0}, f_{1}, f_{2}, f_{02}\right)$,

$$
2(k-1) f_{02}-k(k+1) f_{2}+\left(k^{2}-3 k+4\right) f_{1}-k(k-3) f_{0} \leq 4\left(\begin{array}{c}
f_{0} \\
2
\end{array}\right) .
$$

Equality holds only if every 3-face of $P$ has $k$ or $k+1$ vertices, every two vertices of $P$ lie on a common 2-face, and each 3-face of $P$ is (combinatorially equivalent to) either the pyramid with a $(k-1)$-gon base or the pyramid with a $k$-gon base, or in the case when $k=3$, also the triangular prism.

Proof. For a 4-polytope $P$,

$$
\begin{aligned}
\left(\begin{array}{c}
f_{0} \\
2
\end{array}\right) & \geq m_{1}+m_{2}+m_{3} \\
& =f_{1}+\sum_{\substack{F \in F(P) \\
\operatorname{dim} F=2}} m_{2}(F)+\sum_{\substack{G \in F(P) \\
\operatorname{dim} G=3}} m_{3}(G) \\
& =f_{1}+\sum_{\substack{G \in F(P) \\
\operatorname{dim} G=3}}\left(\frac{1}{2} m_{2}(G)+m_{3}(G)\right) \\
& \geq f_{1}+\frac{1}{2} \sum_{\substack{G \in F(P) \\
\operatorname{dim} G=3}}\left\{k f_{0}(G)-f_{1}(G)-\left(\begin{array}{c}
k+1 \\
2
\end{array}\right)\right\} \\
& =f_{1}+\frac{1}{2}\left\{k f_{03}-f_{13}-\left(\begin{array}{c}
k+1 \\
2
\end{array}\right) f_{3}\right\} .
\end{aligned}
$$

The analysis of equality is similar to that for Theorem 2.1.

The following is immediate by a discriminant consideration.

\section{Corollary 2.6.}

$\left(2 f_{02}-f_{2}-3 f_{1}+3 f_{0}\right)^{2}-8\left(f_{2}-f_{1}+f_{0}\right)\left[2\left(\begin{array}{c}f_{0} \\ 2\end{array}\right)+f_{02}-2 f_{1}\right] \leq\left(f_{2}-f_{1}+f_{0}\right)^{2}$.

We finish this section by giving the duals of the above results.

Theorem 2.1*. For any integer $k$,

$$
(k-1) f_{02}-\left(\begin{array}{c}
k+1 \\
2
\end{array}\right) f_{1}+f_{2} \leq\left(\begin{array}{c}
f_{0}-f_{1}+f_{2} \\
2
\end{array}\right) .
$$

Equality holds only if $k=2,3$, every two facets of $P$ contain a common 1-face, and each vertex figure of $P$ is (combinatorially equivalent to) either the tetrahedron, the pyramid with a square base, or the double pyramid over a triangle.

Corollary 2.2*. For any integer $k \neq 2,3$,

$$
(k-1) f_{02}-\left(\begin{array}{c}
k+1 \\
2
\end{array}\right) f_{1}+f_{2}+1 \leq\left(\begin{array}{c}
f_{0}-f_{1}+f_{2} \\
2
\end{array}\right) .
$$


Corollary 2.3*.

$$
f_{02}\left(f_{02}-3 f_{1}\right) \leq 2 f_{1}\left[\left(\begin{array}{c}
f_{0}-f_{1}+f_{2} \\
2
\end{array}\right)-f_{2}\right] \text {. }
$$

Theorem 2.5*. For any integer $k$,

$$
2(k-1) f_{02}-4 k f_{1}+4 f_{2}-k(k-3) f_{0} \leq 4\left(\begin{array}{c}
f_{0}-f_{1}+f_{2} \\
2
\end{array}\right) .
$$

Equality holds only if each vertex lies on exactly $k$ or $k+1$ facets, every two facets of $P$ contain a common 1-face, and each vertex figure of $P$ is (combinatorially equivalent to) either a pyramid with a $(k-1)$-gon base or a pyramid with a $k$-gon base, or in the case of $k=3$, also the double pyramid over a triangle.

\section{Corollary 2.6*.}

$$
\left(2 f_{02}-4 f_{1}+3 f_{0}\right)^{2}-8 f_{0}\left[2\left(\begin{array}{c}
f_{0}-f_{1}+f_{2} \\
2
\end{array}\right)+f_{02}-2 f_{2}\right] \leq f_{0}^{2} .
$$

For references, these inequalities, expressed in terms of $\left(f_{0}, f_{1}, f_{3}, f_{03}\right)$, are also collected in Appendix C.

\section{Relative Strengths of the Inequalities}

It is natural to ask if there are any redundancies in our lists of inequalities. To begin, we note that despite the ranges of $k$ indicated in these results, the only cases of interest have $k \geq 3$. Henceforth, we assume this restriction on $k$.

We discuss two sets of comparisons in this order: (A) Theorem 2.5 within itself and with Bayer's (9); and (B) Corollary 2.2 within itself and with Bayer's (7).

\section{(A) Theorem 2.5 within Itself and with Bayer's (9)}

For any integer $k$, we define

$$
\begin{aligned}
\xi(k) & =\frac{1}{4}\left\{2(k-1) f_{02}-k(k+1) f_{2}+\left(k^{2}-3 k+4\right) f_{1}-k(k-3) f_{0}\right\} \\
& =\frac{1}{4}\left\{\left(-f_{2}+f_{1}-f_{0}\right) k^{2}+\left(2 f_{02}-f_{2}-3 f_{1}+3 f_{0}\right) k+2\left(-f_{02}+2 f_{1}\right)\right\} .
\end{aligned}
$$

As well, let $B_{9}=f_{02}-4 f_{2}+3 f_{1}-2 f_{0}$.

It is easy to see that $B_{9} \geq \xi(3)$ always, and for quasi-simplicial polytopes, $B_{9} \geq \xi(4)$. However, for many 4-polytopes, we have $B_{9}<\xi(4)$. In fact, we can analyze the proofs given here and given in [1] along this line. Bayer's proof of $B_{9}$ relies on the lemma that says that for 3-polytopes $G$ with at least seven vertices, $m_{2}(G)+m_{3}(G) \geq 2 f_{0}(G)-8$. The argument makes use of the creation of a simplicial 3-polytope by adding extra edges 
to $G$. However, the essence of the approximation may be appreciated even better by a pure, elementary algebraic identity, namely,

$m_{2}(G)+m_{3}(G)=\left(2 f_{1}(G)-3 f_{2}(G)\right)+\frac{1}{2}\left(f_{0}(G)-4\right)\left(f_{0}(G)-7\right)+\left(2 f_{0}(G)-8\right)$.

Thus, Bayer's lower bound comes from dropping $\frac{1}{2}\left(f_{0}(G)-4\right)\left(f_{0}(G)-7\right)$ when there are at least seven vertices, and dropping $2 f_{1}(G)-3 f_{2}(G)$, which is an indicator of the degree to which $G$ deviates from being simplicial. In particular, the lower bound is a fine one only when $G$ is very close to being simplicial. Consequently, Bayer's (9) is good only when $P$ is close to being quasi-simplicial. Our $\xi(4)$, on the other hand, relies on dropping from

$$
m_{2}(G)+m_{3}(G)=\frac{1}{2}\left(f_{0}(G)-4\right)\left(f_{0}(G)-5\right)+4 f_{0}(G)-f_{1}(G)-10
$$

the non-negative term $\frac{1}{2}\left(f_{0}(G)-4\right)\left(f_{0}(G)-5\right)$. In particular, its strength is not diminished when $P$ deviates from being quasi-simplicial. The same can be said of $\xi(k)$ for every $k \geq 4$. In particular, unless $P$ is "almost" quasi-simplicial, $\xi(5)$ is always greater than $B_{9}$. For instance, if $P$ is the pyramid over the 3-cube, $B_{9}=30, \xi(4)=31$, and $\xi(5)=32.5$.

We can go further and ask about how $\xi(k), k \geq 4$, compare with one another. To this end, let $P Q$ denote the pyramid over the 3-polytope $Q$, let $\Pi Q$ denote the prism over the base $Q$, and consider the following polytopes:

$P_{1}=P Q$, where $Q$ is the triangular prism,

$P_{2}=P Q$, where $Q$ is the 3-cube,

$P_{3}=\Pi Q$, where $Q$ is the icosahedron,

$P_{4}=P Q$, where $Q$ is the dodecahedron,

$P_{5}=\Pi Q$, where $Q$ is the 3-cube with one corner cut off,

$P_{6}=\Pi Q$, where $Q$ is the 3-cube with two corners cut off,

$P_{7}=\Pi Q$, where $Q$ is the 3-cube with the four corners that correspond to the vertices of an embedded regular tetrahedron cut off,

$P_{8}=\Pi Q$, where $Q$ is the dodecahedron.

The values of $B_{9}$ and $\xi\left(k_{0}\right)=\max \{\xi(k): k \geq 4, k$ an integer $\}$ are shown:

\begin{tabular}{lccrrrrrr}
\hline & $P_{1}$ & $P_{2}$ & $P_{3}$ & $P_{4}$ & $P_{5}$ & $P_{6}$ & $P_{7}$ & $P_{8}$ \\
\hline$B_{9}$ & 20 & 30 & 128 & 90 & 84 & 104 & 144 & 184 \\
$\xi\left(k_{0}\right)$ & 20.5 & 32.5 & 153 & 115 & 138 & 183 & 278 & 378 \\
$k_{0}$ & 4 & 5 & 6 & 7 & 8 & 9 & 10 & 11 \\
\hline
\end{tabular}

These examples, for one thing, show that none of the inequalities on the list (with $k \geq 4$ ) is stronger than all the rest. The question is whether there is a finite subset of the list so that taken together they are stronger than all the remaining ones. The examples above show that such a subset, if it exists, should include $\xi(k), 4 \leq k \leq 11$. We found these examples inside the classes of pyramids and prisms. Unfortunately, the formation of pyramids and prisms cannot lead us too far. Indeed, for pyramids, the maximum of $\xi(k)$ is attained at some $k \leq 8$, and for prisms, at some $k \leq 15$. The following are straightforward to 
check: The quadratic expression $\xi(k), k$ integer, is maximized at $k=\lfloor\mu\rfloor$ or $\lfloor\mu\rfloor+1$, where

$$
\mu=\frac{2 f_{02}-f_{2}-3 f_{1}+3 f_{0}}{2 f_{3}} .
$$

For pyramids $P Q$, this reduces to

$$
\mu=\frac{6 f_{1}(Q)-f_{2}(Q)+3}{2\left(1+f_{2}(Q)\right)} \leq \frac{17 f_{2}(Q)-33}{2 f_{2}(Q)+2}<\frac{17}{2} ;
$$

and for prisms $\Pi Q$, it reduces to

$$
\mu=\frac{12 f_{0}(Q)+7 f_{2}(Q)-18}{2 f_{2}(Q)+4} \leq \frac{31 f_{2}(Q)-66}{2 f_{2}(Q)+4}<\frac{31}{2} .
$$

Nevertheless, we would like to make this conjecture:

Conjecture 3.1. For any $k_{0} \geq 4$, there is a 4-polytope for which $\xi\left(k_{0}\right)>\xi(3)$, and $\xi\left(k_{0}\right)>\xi(k)$ for all $k \geq 4, k \neq k_{0}$.

(B) Corollary 2.2 within Itself and with Bayer's (7)

For any integer $k \geq 3$, we define

$$
\psi(k)=(k-1) f_{02}-\left(\begin{array}{c}
k+1 \\
2
\end{array}\right) f_{2}+f_{1},
$$

and for $k \geq 4, \psi^{+}(k)=\psi(k)+1$. We also write $B_{7}=2\left(f_{02}-3 f_{2}\right)+f_{1}$. Then as mentioned as above, $B_{7}=\psi(3)$. Now, how do $\psi(3)$ and $\psi^{+}(k), k \geq 4$, compare with each other? We do not have a complete answer, but note that if $P$ is the 4 -simplex, then

$$
B_{7}=\psi(3)=10>\psi^{+}(k)
$$

for all $k \geq 4$. Thus, none of the cases $k \geq 4$ is stronger than $B_{7}$. On the other hand, $B_{7}$ is not stronger than $\psi^{+}(4)$ as the prism over the dodecahedron shows. Examples of 4-polytopes for which the maximum of $\psi^{+}(k)$ is attained at some $k>4$ seem to be harder to construct. Prisms over a 3-polytope always maximize $\psi^{+}(k)$ at $k=3$ or 4 . Neverthelss, we still want to make the following conjecture:

Conjecture 3.2. For any $k_{0} \geq 4$, there is a 4-polytope for which $\psi^{+}\left(k_{0}\right)>\psi(3)$, and $\psi^{+}\left(k_{0}\right)>\psi^{+}(k)$ for all $k \geq 4, k \neq k_{0}$.

\section{Projections onto the $f$-Vector Space and Fatness}

We can take our newly found nonlinear inequalities, and project them onto the $f$-vector space by using Bayer's (1)-(6). We omit the proofs. 
Theorem 4.1. For any integer $k \geq 3$ :

(B1) \&(2.5) $\quad\left(k^{2}-3 k+4\right) f_{1} \leq 4\left(\begin{array}{c}f_{0} \\ 2\end{array}\right)+2\left(\begin{array}{c}k-2 \\ 2\end{array}\right) f_{2}+k(k-3) f_{0}$.

(B1) $\&(2.1)^{*}(3 k-2) f_{2} \leq\left(\begin{array}{c}k+1 \\ 2\end{array}\right) f_{1}+\left(\begin{array}{c}f_{0}-f_{1}+f_{2} \\ 2\end{array}\right)$.

(B1) \&(2.2)* $(3 k-2) f_{2} \leq\left(\begin{array}{c}k+1 \\ 2\end{array}\right) f_{1}+\left(\begin{array}{c}f_{0}-f_{1}+f_{2} \\ 2\end{array}\right)-1$, if $k \geq 4$.

(B1)\&(2.5)* $2(3 k-1) f_{2} \leq 4\left(\frac{f_{0}-f_{1}+f_{2}}{2}\right)+4 k f_{1}+k(k-3) f_{0}$.

(B2) $\&(2.1) \quad(3 k-2) f_{1} \leq\left(\begin{array}{c}k+1 \\ 2\end{array}\right) f_{2}+\left(\begin{array}{c}f_{0} \\ 2\end{array}\right)$

(B2)\&(2.2) $\quad(3 k-2) f_{1} \leq\left(\begin{array}{c}k+1 \\ 2\end{array}\right) f_{2}+\left(\begin{array}{c}f_{0} \\ 2\end{array}\right)-1$, if $k \geq 4$.

(B2)\&(2.5) $\quad\left(k^{2}+3 k-2\right) f_{1} \leq 4\left(\begin{array}{c}f_{0} \\ 2\end{array}\right)+k(k+1) f_{2}+k(k-3) f_{0}$.

(B2)\&(2.5)* $2(k-3) f_{1}+4 f_{2} \leq 4\left(f_{0}-f_{1}+f_{2}\right)+k(k-3) f_{0}$.

(B3) \&(2.1) $4(k-1) f_{0} \leq\left(\begin{array}{c}f_{0} \\ 2\end{array}\right)+\left(\begin{array}{c}k-2 \\ 2\end{array}\right) f_{2}+(k-2) f_{1}+10(k-1)$.

(B3) $\&(2.2) \quad 4(k-1) f_{0} \leq\left(\begin{array}{c}f_{0} \\ 2\end{array}\right)+\left(\begin{array}{c}k-2 \\ 2\end{array}\right) f_{2}+(k-2) f_{1}+10 k-11$, if $k \geq 4$.

(B3) $\&(2.5) \quad 2\left(\begin{array}{c}k-2 \\ 2\end{array}\right) f_{1} \leq 4\left(\begin{array}{c}f_{0} \\ 2\end{array}\right)+2\left(\begin{array}{c}k-2 \\ 2\end{array}\right) f_{2}+\left(k^{2}-11 k+8\right) f_{0}+20(k-1)$.

(B3) $\&(2.1)^{*}(3 k-2) f_{2}+4(k-1) f_{0} \leq \frac{1}{2}\left(k^{2}+3 k-2\right) f_{1}+\left(\begin{array}{c}f_{0}-f_{1}+f_{2} \\ 2\end{array}\right)+10(k-1)$.

(B3) \&(2.2)* $(3 k-2) f_{2}+4(k-1) f_{0} \leq \frac{1}{2}\left(k^{2}+3 k-2\right) f_{1}+\left(\begin{array}{c}f_{0}-f_{1}+f_{2} \\ 2\end{array}\right)+10 k-11$, if $k \geq 4$.

(B3) $\&(2.5)^{*} 2(3 k-1) f_{2} \leq 4\left(\frac{f_{0}-f_{1}+f_{2}}{2}\right)+2(3 k-1) f_{1}+\left(k^{2}-11 k+8\right) f_{0}+20(k-1)$.

Remarks. 1. We do not list (B1)\&(2.1), (B1)\&(2.2), (B2)\&(2.1)*, and (B2)\&(2.2)*, for they are trivial.

2. We note that (B2) is the dual of (B1), and so $(\mathrm{B} i) \&(2 . j)$ is the dual of $(\mathrm{B}(3-$ $i)) \&(2 . j)^{*}$ for $i=1,2 ; j=1,2,5$. As well, (B3) is self-dual. So, (B3)\&(2.j) is the dual of (B3)\&(2.j)* for $j=1,2,5$.

3. These same inequalities are written in terms of $\left(f_{0}, f_{1}, f_{3}, f_{03}\right)$ in Appendix D.

As a result of Theorem 4.1 (B1)\&(2.5), and (B2)\&(2.5)* we have

Corollary 4.2. For any integer $k \geq 3$,

$$
\begin{aligned}
f_{1} & \leq \frac{1}{k} f_{0}^{2}+f_{0}+\frac{k}{2} f_{3}, \\
f_{1} & \leq \frac{1}{k} f_{3}^{2}+\frac{1}{k} f_{0}+\frac{k}{2} f_{0} .
\end{aligned}
$$

As well, if $n=\max \left\{f_{0}, f_{3}\right\}$ and $k \geq 3$, then

$$
f_{1} \leq\left\lfloor\frac{1}{k} n^{2}+\frac{k-1}{2} n\right\rfloor \text {. }
$$

Consequently, $f_{1}=O\left(n^{3 / 2}\right)$.

Remark. This last result, although not strong, does fare better than

$$
f_{1} \leq\left\lfloor\frac{n^{2}+3 n}{4}\right\rfloor
$$


mentioned in [6], as can be seen from its asymptotic behaviour. As a result, then, Theorem 4.1 also eventually eliminates the infinite sequence of "potential" vectors $\left(f_{0}, f_{1}, f_{3}, f_{03}\right)$,

$$
\begin{array}{ll}
\left(4 k, 4 k^{2}+3 k, 4 k, 4 k^{2}+11 k\right) & \text { for } \quad k \geq 2, \\
\left(4 k+1,4 k^{2}+5 k+1,4 k+1,4 k^{2}+13 k+3\right) & \text { for } \quad k \geq 1, \\
\left(4 k+2,4 k^{2}+7 k+2,4 k+2,4 k^{2}+15 k+6\right) & \text { for } \quad k \geq 2, \\
\left(4 k+2,4 k^{2}+7 k+2,4 k+2,4 k^{2}+15 k+7\right) & \text { for } \quad k \geq 1, \\
\left(4 k+3,4 k^{2}+9 k+4,4 k+3,4 k^{2}+17 k+10\right) & \text { for } \quad k \geq 2, \\
\left(4 k+3,4 k^{2}+9 k+4,4 k+3,4 k^{2}+17 k+11\right) & \text { for } \quad k \geq 1,
\end{array}
$$

constructed there, for as $k$ gets large, these will no longer be "potential". It should be noted, however, that our result still does not beat Edelsbrunner and Sharir's bound $f_{1}=O\left(n^{4 / 3}\right)$, which has also been mentioned by Höppner and Ziegler [6].

\section{Conjectures and Open Problems}

1. With regard to Conjectures 3.1 and 3.2, the present author is betting on the prolificacy of counter-intuitive scenarios in four dimensions. If these conjectures turn out to be false, it could be, at least partially, due to the fact that 3-polytopes cannot have too many facets of too many sides, as shown by Roudneff [9].

2. On the new list, four inequalities stand out as having a particularly nice form:

$$
\begin{aligned}
(2.3) \quad f_{02}\left(f_{02}-3 f_{2}\right) & \leq 2 f_{2}\left[\left(\begin{array}{c}
f_{0} \\
2
\end{array}\right)-f_{1}\right] . \\
(2.3)^{*} & f_{02}\left(f_{02}-3 f_{1}\right) \leq 2 f_{1}\left[\left(\begin{array}{c}
f_{3} \\
2
\end{array}\right)-f_{2}\right] . \\
\left(2.6^{\prime}\right) \quad f_{03}\left(f_{03}-3 f_{3}\right) & \leq 4 f_{3}\left[\left(\begin{array}{c}
f_{0} \\
2
\end{array}\right)-f_{0}\right] . \\
\left(2.6^{\prime}\right)^{*} & f_{03}\left(f_{03}-3 f_{0}\right) \leq 4 f_{0}\left[\left(\begin{array}{c}
f_{3} \\
2
\end{array}\right)-f_{3}\right] .
\end{aligned}
$$

These are the consequences of collapsing a sequence of inequalities by a discriminant calculation. We conjecture that each of them indeed implies the corresponding sequence of inequalities.

3. Can we find nice geometric interpretations and explanations for the above four cubic inequalities?

\section{Appendix A. Flag $f$-Vector Components in Terms of $\left(f_{0}, f_{1}, f_{2}, f_{02}\right)$, and $\left(f_{0}, f_{1}, f_{3}, f_{03}\right)$}

In terms of $\left(f_{0}, f_{1}, f_{2}, f_{02}\right)$, these are from [1]:

$$
\begin{array}{lll}
f_{0}=\text { basic; } & f_{02}=\text { basic; } & f_{012}=2 f_{02} ; \\
f_{1}=\text { basic; } & f_{03}=2 f_{0}-2 f_{1}+f_{02} ; & f_{013}=2 f_{02} ; \\
f_{2}=\text { basic; } & f_{12}=f_{02} ; & f_{023}=2 f_{02} ; \\
f_{3}=f_{0}-f_{1}+f_{2} ; & f_{13}=f_{02} ; & f_{123}=2 f_{02} ; \\
f_{01}=2 f_{1} ; & f_{23}=2 f_{2} ; & f_{0123}=4 f_{02} .
\end{array}
$$


In terms of $\left(f_{0}, f_{1}, f_{2}, f_{03}\right)$ :

$$
\begin{array}{lll}
f_{0}=\text { basic; } & f_{02}=-2 f_{0}+2 f_{1}+f_{03} ; & f_{012}=-4 f_{0}+4 f_{1}+2 f_{03} ; \\
f_{1}=\text { basic } ; & f_{03}=\text { basic } ; & f_{013}=-4 f_{0}+4 f_{1}+2 f_{03} ; \\
f_{2}=-f_{0}+f_{1}+f_{3} ; & f_{12}=-2 f_{0}+2 f_{1}+f_{03} ; & f_{023}=-4 f_{0}+4 f_{1}+2 f_{03} ; \\
f_{3}=\text { basic; } & f_{13}=-2 f_{0}+2 f_{1}+f_{03} ; & f_{123}=-4 f_{0}+4 f_{1}+2 f_{03} ; \\
f_{01}=2 f_{1} ; & f_{23}=-2 f_{0}+2 f_{1}+2 f_{3} ; & f_{0123}=-8 f_{0}+8 f_{1}+4 f_{03} .
\end{array}
$$

\section{Appendix B. Ten Inequalities in Terms of $\left(f_{0}, f_{1}, f_{3}, f_{03}\right)$}

The labels for these inequalities are those in [6], and the inequalities are (B1)-(B10), in that order:

(3.1) Linear inequalities:

$$
\begin{aligned}
& \text { (3.1.1) }-f_{0}+f_{1}+3 f_{3}-f_{03} \leq 0 . \\
& \text { (3.1.2) } 2 f_{0}+f_{1}-f_{03} \leq 0 \\
& \text { (3.1.3) } 3 f_{0}+3 f_{3}-f_{03} \leq 10 \\
& \text { (3.1.4) } 4 f_{0}-4 f_{1}+f_{03} \leq 0 \\
& \text { (3.1.5) } f_{0} \geq 5 \\
& \text { (3.1.6) } f_{3} \geq 5
\end{aligned}
$$

(3.2) Nonlinear inequalities:

$$
\begin{aligned}
& \text { (3.2.1) } 2 f_{0}-f_{1}-6 f_{3}+2 f_{03} \leq\left(\begin{array}{c}
f_{0} \\
2
\end{array}\right) . \\
& \text { (3.2.2) }-5 f_{0}-f_{1}+f_{3}+2 f_{03} \leq\left(\begin{array}{c}
f_{3} \\
2
\end{array}\right) . \\
& \text { (3.2.3) } f_{1}-4 f_{3}+f_{03} \leq\left(\begin{array}{c}
f_{0} \\
2
\end{array}\right) . \\
& \text { (3.2.4) }-5 f_{0}+f_{1}+f_{3}+f_{03} \leq\left(\begin{array}{c}
f_{3} \\
2
\end{array}\right) .
\end{aligned}
$$

\section{Appendix C. New Nonlinear Inequalities for Flag $f$-Vectors}

With known redundancies removed, we list the newly found inequalities in terms of $\left(f_{0}, f_{1}, f_{2}, f_{02}\right)$ :

(2.2) For any integer $k \geq 4,(k-1) f_{02}-\left(\begin{array}{c}k+1 \\ 2\end{array}\right) f_{2}+f_{1}+1 \leq\left(\begin{array}{c}f_{0} \\ 2\end{array}\right)$.

(2.3) $f_{02}\left(f_{02}-3 f_{2}\right) \leq 2 f_{2}\left[\left(\begin{array}{c}f_{0} \\ 2\end{array}\right)-f_{1}\right]$.

(2.5) For any integer $k, 2(k-1) f_{02}-k(k+1) f_{2}+\left(k^{2}-3 k+4\right) f_{1}-k(k-3) f_{0} \leq 4\left(\begin{array}{c}f_{0} \\ 2\end{array}\right)$.

(2.6) $\left(2 f_{02}-f_{2}-3 f_{1}+3 f_{0}\right)^{2}-8\left(f_{2}-f_{1}+f_{0}\right)\left[2\left(\begin{array}{c}f_{0} \\ 2\end{array}\right)+f_{02}-2 f_{1}\right] \leq\left(f_{2}-\right.$ $\left.f_{1}+f_{0}\right)^{2}$.

$(2.2)^{*}$ For any integer $k \geq 4,(k-1) f_{02}-\left(\begin{array}{c}k+1 \\ 2\end{array}\right) f_{1}+f_{2}+1 \leq\left(\begin{array}{c}f_{0}-f_{1}+f_{2} \\ 2\end{array}\right)$.

$(2.3)^{*} f_{02}\left(f_{02}-3 f_{1}\right) \leq 2 f_{1}\left[\left(\begin{array}{c}f_{0}-f_{1}+f_{2} \\ 2\end{array}\right)-f_{2}\right]$.

$(2.5)^{*}$ For any integer $k, 2(k-1) f_{02}-4 k f_{1}+4 f_{2}-k(k-3) f_{0} \leq 4\left(\begin{array}{c}f_{0}-f_{1}+f_{2} \\ 2\end{array}\right)$.

$(2.6)^{*}\left(2 f_{02}-4 f_{1}+3 f_{0}\right)^{2}-8 f_{0}\left[2\left(\frac{f_{0}-f_{1}+f_{2}}{2}\right)+f_{02}-2 f_{2}\right] \leq f_{0}^{2}$. 
Likewise, in terms of $\left(f_{0}, f_{1}, f_{3}, f_{03}\right)$, we have:

(2.2') For any integer $k \geq 4,(k-1) f_{03}+\frac{1}{2}\left(k^{2}-3 k+4\right) f_{0} \leq\left(\begin{array}{c}f_{0} \\ 2\end{array}\right)+\left(\begin{array}{c}k+1 \\ 2\end{array}\right) f_{3}+$ $\left(\begin{array}{c}k-1 \\ 2\end{array}\right) f_{1}-1$.

$\left(2.3^{\prime}\right) \quad\left(f_{03}-2 f_{0}+2 f_{1}\right)\left(f_{03}+f_{0}-f_{1}-3 f_{3}\right) \leq 2\left(f_{3}+f_{1}-f_{0}\right)\left[\left(\begin{array}{c}f_{0} \\ 2\end{array}\right)-f_{1}\right]$.

$\left(2.5^{\prime}\right) \quad$ For any integer $k,(k-1) f_{03} \leq f_{0}\left(f_{0}-3\right)+\left(\begin{array}{c}k+1 \\ 2\end{array}\right) f_{3}$.

(2.6 $) \quad f_{03}\left(f_{03}-3 f_{3}\right) \leq 4 f_{3}\left[\left(\begin{array}{c}f_{0} \\ 2\end{array}\right)-f_{0}\right]$.

$\left(2.2^{\prime}\right)^{*}$ For any integer $k \geq 4,(k-1) f_{03}+f_{3}+1 \leq\left(\begin{array}{c}f_{3} \\ 2\end{array}\right)+(2 k-1) f_{0}+\left(\begin{array}{c}k-1 \\ 2\end{array}\right) f_{1}$.

$\left(2.3^{\prime}\right)^{*}\left(f_{03}-2 f_{0}+2 f_{1}\right)\left(f_{03}-2 f_{0}-f_{1}\right) \leq 2 f_{1}\left[\left(\begin{array}{c}f_{3} \\ 2\end{array}\right)+f_{0}-f_{1}-f_{3}\right]$.

$\left(2.5^{\prime}\right)^{*}$ For any integer $k,(k-1) f_{03}+2 f_{3} \leq 2\left(\begin{array}{c}f_{3} \\ 2\end{array}\right)+\left(\begin{array}{c}k+1 \\ 2\end{array}\right) f_{0}$.

$\left(2.6^{\prime}\right)^{*} f_{03}\left(f_{03}-3 f_{0}\right) \leq 4 f_{0}\left[\left(\begin{array}{c}f_{3} \\ 2\end{array}\right)-f_{3}\right]$.

\section{Appendix D. New Nonlinear Inequalities for $f$-Vectors}

Theorem 4.1, in terms of $\left(f_{0}, f_{1}, f_{3}, f_{03}\right)$, reads:

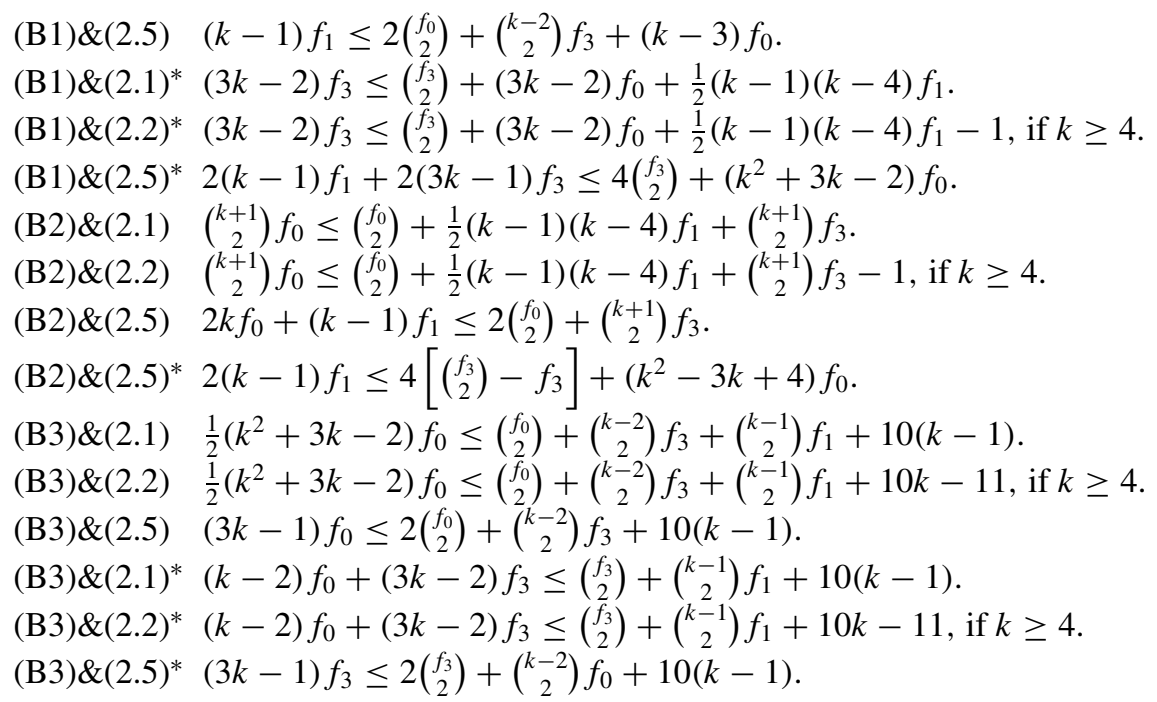

\section{Acknowledgments and Addenda}

The author thanks the two referees for providing valuable comments and criticisms, which have helped the author correct errors and typos and clarify a few points. One referee also disproved Corollary 3.2 by showing that $\psi^{+}(k)$ is always maximized at $k=3,4$, or 5 . The same referee also obtained an improvement of the second inequality in Corollary 4.2. 


\section{References}

1. Margaret Bayer, The extended f-vectors of 4-polytopes, J. Combin. Theory Ser. A 44(1) (1987), 141-151.

2. Margaret Bayer and Louis J. Billera, Generalized Dehn-Sommerville relations for polytopes, spheres and Eulerian partially ordered sets, Invent. Math. 79(1) (1985), 143-157.

3. Louis J. Billera and Anders Björner, Face numbers of polytopes and complexes, in Jacob E. Goodman and Joseph O'Rourke, eds., Handbook of Discrete and Computational Geometry, 2nd edn., CRC Press, Boca Raton, FL, 2004, pp. 407-430.

4. Richard Ehrenborg, Lifting inequalities for polytopes, Adv. Math. 193 (2005), 205-222.

5. Branko Grünbaum, Convex Polytopes, 2nd edn., Springer-Verlag, New York, 2003.

6. Andrea Höppner and Günter M. Ziegler, A census of flag-vectors of 4-polytopes, in Gil Kalai and Günter M. Ziegler, eds., Polytopes-Combinatorics and Computation, Birkhäuser, Basel, 2000, pp. 105-110.

7. Gil Kalai, Peter Kleinschmidt, and Günter Meisinger, Flag numbers and FLAGTOOL, in Gil Kalai and Günter M. Ziegler, eds., Polytopes—Combinatorics and Computation, Birkhäuser, Basel, 2000, pp. 75-103.

8. Andreas Paffenholz, Construction for posets, lattices, and polytopes, Dissertation, Berlin, 2005.

9. Jean-Pierre Roudneff, An inequality for 3-polytopes, J. Combin. Theory Ser. B 42 (1987), 156-166.

10. Catherine Stenson, Relationships among flag f-vector inequalities for polytopes, Discrete Comput. Geom. 31(2) (2004), 257-273.

11. Günter M. Ziegler, Questions about polytopes, in Björn Engquist and Wilfred Schmid, eds., Mathematics Unlimited -2001 and Beyond, Springer-Verlag, New York, 2001, pp. 1195-1211.

12. Günter M. Ziegler, Projected products of polygons, arXiv:math.MG/0407042v1, 3 July 2004.

Received September 29, 2005, and in revised form September 1, 2006. Online publication March 2, 2007. 